\title{
OTIONOMICS
}

Revista de economía, empresa y sociedad

Dossier «Marketing digital: revolucionando el consumo y la sociedad»

MARKETING MÓVIL: CLAVE EN LA ERA DIGITAL

\section{Movilidad, aquello que lo transforma todo}

\section{Kavier Folguera Obiol}

Profesor colaborador del máster universitario de Marketing Digital (UOC).

Director de la agencia digital Advertis

RESUMEN El marketing móvil es un ámbito del marketing digital que se está reinterpretando constantemente debido al imparable avance de unos dispositivos inteligentes, nacidos hace poco más de diez años, que tienen poco que ver con los teléfonos móviles convencionales aparecidos en los años noventa del pasado siglo. Precisamente, como la movilidad interconectada está cambiando los hábitos humanos, el resto de áreas de conocimiento del marketing digital están adaptándose a una nueva realidad que todavía no dispone de patrones de comportamiento conocidos. Es tan grande el impacto de la movilidad en los entornos del marketing digital, como los que representan la publicidad, los buscadores, las redes sociales, el marketing por correo electrónico o el marketing promocional, que todos ellos se han ido transformando en detrimento de la tradicional navegación y las funcionalidades vinculadas al ordenador de sobremesa. Por lo tanto, ya estamos alcanzando aquel momento en que la movilidad digital forma parte implícita en sí misma de la definición del marketing, y se podría decir que el concepto independiente de marketing móvil tiene los días contados.

PALABRAS CLAVE movilidad; app; portabilidad; geolocalización; personalización; privacidad; instantaneidad; automatización; inteligencia artificial; realidad aumentada; m-commerce; patrones de comportamiento

\section{Mobility transforms everything}

ABSTRACT Mobile marketing is a field of digital marketing constantly being reinterpreted due to the unstoppable progress of smart devices, founded little more than ten years ago, which have little to do with the conventional mobile phones that appeared in the nineteen nineties. In the same manner that interconnected mobility is changing human habits, the rest of the areas in digital marketing knowledge are adapting to a new reality which does not yet have known behavioural patterns. Mobility has an immense impact on digital marketing spheres, such as advertising, search engines, social networks, email marketing and promotional marketing. All these have been transforming, to the detriment of traditional browsing and functionalities linked to desktop computers. We are therefore reaching that moment in which digital mobility itself forms an implicit part of the definition of marketing. We could say that the concept independent of mobile marketing has its days numbered.

KEYWORDS mobility; app; portability; geolocation; personalisation; privacy; instantaneousness; automation; artificial intelligence; augmented reality; m-commerce; behavioural patterns 


\section{Introducción: la movilidad interconectada}

Dentro de la revolución digital del siglo XXI, no podía quedar al margen una de las características que definen mejor la esencia humana: la capacidad de movilidad de los individuos. Solo en una década se está modificando el modo en que las personas se relacionan, se comunican, se informan o adquieren bienes y conocimientos, y todo gracias a la aparición de dispositivos móviles inteligentes. Unos dispositivos portables que permiten una conectividad impensable hace tan solo veinte años, donde el individuo forma parte de comunidades y disfruta de los vínculos sociales de modo casi permanente, esté donde esté y de forma simultánea. Así pues, la existencia de las redes sociales y las comunidades online, las aplicaciones móviles y los nuevos hábitos de consumo han convertido los dispositivos móviles en omnipresentes en nuestra sociedad (Shankar et al., 2010).

La magnitud del cambio debido a la movilidad y sus efectos en el desarrollo del marketing vaticinan una próxima transformación de la economía y la sociedad nunca vista hasta el momento, a una velocidad inaudita. El nacimiento de internet no será propiamente el precursor del cambio, sino su adaptación a las necesidades humanas (Drucker, 1999). Haber instalado internet en un teléfono móvil está siendo tan importante en la historia de la humanidad como haber situado la fuerza de la máquina de vapor sobre unas vías férreas o haber reproducido masivamente ejemplares de la Biblia con la nueva imprenta inventada en el siglo xv. Así pues, la movilidad será clave en la definitiva defunción de la edad contemporánea y el nacimiento de la prometedora edad digital.

El entorno tecnológico de hoy nos permite, de manera cotidiana, percibir que las empresas llevan actividades persuasivas para retener nuestro interés, gracias al conocimiento que pueden tener de nosotros, de nuestros hábitos de vida y de nuestra movilidad. Con los dispositivos móviles, los comerciantes son capaces de mostrarnos personas virtuales proyectadas con hologramas en los comercios mediante la realidad aumentada o anuncios con ofertas personalizadas cuando paseamos por la calle, o bien, como por arte de magia, pueden mandarnos mensajes adaptados a nosotros. Precisamente por ello, y debido al hecho de considerarnos el centro de universo, del mismo modo que sucede en muchas invenciones humanas, no todo es positivo. El impacto de la innovación referente a los dispositivos móviles está provocando la aparición de paradojas importantes vinculadas a los derechos a la privacidad de las personas y a la invasión de lo que se considera personal.

\section{El marketing: será móvil, o no será}

En los entornos profesionales y académicos, el marketing móvil se define como la comunicación o la promoción bidireccional o multidireccional entre las marcas y sus públicos mediante dispositivos y tecnologías móviles (Shankar, 2009). Estos dispositivos tienden a ser personales y están adaptados a las necesidades de cada individuo. Por medio de estos instrumentos, cada persona tiene la capacidad de organizar el tipo de conectividad y el uso de los aparatos que más le conviene, pero también recibe, del resto de la comunidad, mensajes y contenidos que pueden estar relacionados con su geolocalización, su perfil personal o el contexto social y personal donde se encuentra en cada momento. Esta potencia comunicativa está siendo utilizada por las organizaciones para enfocar mejor sus estrategias de marketing, lo que está cambiando las pautas de actuación que hasta ahora se llevaban a cabo.

En realidad, los dispositivos móviles pueden considerarse como un factor de transformación del resto de las herramientas digitales y, en consecuencia, el marketing móvil se configura como una macroherramienta digital de orden superior, donde cada una de las herramientas del marketing digital ya existentes adquieren una dimensión multiplicadora.

Las redes sociales, que vieron la luz en ordenadores de sobremesa conectados a internet, han adquirido con la movilidad una naturaleza casi imprescindible para muchos. El hecho es que se han adaptado a las necesidades de comunicación relativas a la temporalidad y la ubicación, haciéndolo mediante aplicaciones nativas pensadas para resolver funciones específicas relacionadas con el espacio/tiempo de cada persona. Instagram nació a partir de una necesidad comunicativa visual, del mismo modo que pasó en la época prehistórica con las pinturas rupestres (Instazood, 2018). WhatsApp se creó a partir de una necesidad discursiva de todos nosotros que daba voz 
a la espontaneidad y la necesidad de permanente contacto fuéramos donde fuéramos, de una manera simple, segura y rápida (Flora, 2015).

Asimismo, otra herramienta del marketing móvil que ha adquirido una personalidad propia a partir de los dispositivos móviles ha sido el marketing por correo electrónico, marketing por e-mail o marketing de permiso. Si antes de la irrupción de los aparatos móviles los mensajes de correo electrónico eran masivos y solo se consideraban una transposición digital del antiguo correo postal, hoy el envío de mensajes automatizados vinculados a la navegación móvil está provocando un impacto en la rapidez y la personalización de la comunicación que modifica las estrategias de muchas empresas para con sus clientes. En los últimos años, con la recopilación de datos de forma masiva en las redes sociales y en los móviles, los profesionales han cambiado drásticamente el modo en que posicionan el canal de correo electrónico, por la segmentación y el dinamismo que puede ofrecer (Smart Insights, 2013).

Por otra parte, la movilidad está ofreciendo otra perspectiva del marketing de buscadores. Google ha modificado recientemente su algoritmo de organización de enlaces para dar respuesta a la necesidad de búsqueda en movilidad, relegando la importancia que tenían los dispositivos estáticos como, por ejemplo, los ordenadores de sobremesa (Google, 2018). Las campañas de anuncios de búsqueda (SEM) ya pueden tener en cuenta la ubicación y las características de las personas. En paralelo, la clasificación orgánica de las páginas indexadas por los buscadores (SEO) está supeditada a la calidad de los espacios web, en cuanto a su distinción cualitativa móvil. En otras palabras, si un sitio web no se adapta a los requerimientos de calidad exigibles a las pequeñas pantallas y no responde bien a la velocidad de descarga en los dispositivos, no puede aspirar a los lugares preferentes de las listas de indexación relacionadas con las palabras claves que las personas introducimos para encontrar lo que buscamos.

Y si las redes sociales, el marketing por e-mail y los buscadores han mutado debido a la movilidad, la reina de la difusión comercial, la publicidad display, también está adquiriendo una nueva personalidad, poco reconocible sin la naturaleza de la geolocalización, y también, hay que decirlo, los avances de la inteligencia artificial. Dependiendo de la ubicación, el contexto social y geográfico y los intereses de cada individuo, la tecnología ya es capaz de crear y mostrar anuncios casi personalizados que, según las evidencias y el sentido común, pueden aumentar la capacidad de persuasión que tanto esperan los anunciantes de sus campañas publicitarias (Rivillas, 2018). Además, aparte de permitir nuevas dimensiones a la publicidad por su capacidad de absorber las características de la inteligencia artificial, los móviles han forzado el desarrollo de nuevos formatos publicitarios adaptados a los nuevos tiempos. Un ejemplo relevante ha sido la aparición del nuevo formato de vídeos llamado bumper, de solo seis segundos de duración, pensado exclusivamente para los dispositivos móviles (Boni, 2018).

Así pues, no es descabellado pensar que, debido a la transformación e influencia de los dispositivos móviles en las estrategias de marketing digital, la definición natural y actual de marketing móvil, como disciplina aislada o prescindible, puede convertirse en una perogrullada catedralicia en pocos años. Porque el marketing, sea digital o no, o será móvil, o no será. El acceso a la red mediante los dispositivos móviles supera ya en número a la realizada mediante ordenadores (AIMC, 2017) y, por tanto, no hay marcha atrás en la concepción de las nuevas estrategias de marketing que deben dar prioridad a la movilidad.

\section{El impacto del marketing móvil: herramientas y cifras}

El cambio de magnitud del impacto móvil se produjo en 2007 con la aparición del iPhone, que dejaba atrás los teléfonos que utilizaban navegadores de la misma manera que lo hacían los ordenadores de mesa. Una manera de funcionar que no consolidaba el ancho de banda en movilidad (Pérez et al., 2018), pero que alcanzó posteriormente con la aparición de aplicaciones nativas (APP), que elevaron las prestaciones de internet por su encaje en las funcionalidades de los dispositivos. Estas aplicaciones se identifican íntimamente con el Bluetooth, la cámara fotográfica, el wifi, el escáner de códigos QR y, por encima de todo, con el rastro digital producido por GPS (geolocalización). Este conjunto de funcionalidades constituye una amalgama de prestaciones que están cambiando la realidad del uso de la tecnología móvil, como, por ejemplo, la disminución de las tradicionales líneas telefónicas en beneficio de la llamada voz IP, especialmente por el empuje de WhatsApp (ONTSI, 2018). 
Las herramientas que existían antes de la llegada de los dispositivos inteligentes, aunque pueda sorprender, todavía siguen vigentes. Con más de dos décadas en los hombros, los veteranos mensajes cortos (SMS) y, en menor magnitud, el MMS y los códigos QR todavía están presentes en las campañas de los anunciantes y sus estrategias de difusión, entre otras razones, porque así lo desea el cliente. Más del $40 \%$ de los internautas prefieren recibir notificaciones vía mensajes cortos cuando deben ser avisados en compras online o cuando se hacen cargos en sus cuentas bancarias (ONTSI, 2018).

Con todo, más del $70 \%$ de los directivos de empresas anunciantes manifiestan que utilizan herramientas móviles en las actividades de marketing de sus empresas. Por orden de importancia, las primeras posiciones las ocupan el uso de webs responsivas (adaptables a todos los tamaños de pantalla), la publicidad display móvil y el desarrollo de aplicaciones móviles. Es comprensible esta sensibilidad hacia el canal móvil si se tiene presente que, en 2017 en España, un 97\% de los internautas ya disponía de smartphones, superando los 29 millones de dispositivos. El 80\% de jóvenes de entre 18 y 24 años tenía instaladas varias herramientas de mensajería instantánea, por poner solo algunos ejemplos relevantes (IAB, 2017).

\section{Luces y sombras de la movilidad interconectada}

Se podría creer que el avance que ha significado la aparición de estas innovaciones tecnológicas solo trae ventajas, pero nada más lejos de la realidad. Lo cierto es que se están produciendo paradojas importantes en diversos contextos, ya sean de orden personal o individual, a nivel social y también a nivel económico.

La proliferación de los dispositivos y el aumento de su uso están aumentando la inquietud de los usuarios por la invasión a su intimidad. Los dispositivos móviles están considerados aparatos privados donde nadie ajeno a los intereses de su propietario puede inmiscuirse. Dicha acción puede generar sentimiento de usurpación de informaciones que pueden atacar el derecho a la intimidad y la privacidad; unos derechos que están amparados por el ordenamiento jurídico vigente. Todo el mundo conoce el rastro que dejamos mediante la aceptación de galletas (cookies) para poder disfrutar de la navegación por internet. También sabemos que nuestros datos, la auténtica materia prima que sustenta el negocio publicitario de muchos online players, son necesarios para obtener los beneficios de la red, en muchos servicios que pensamos que son gratuitos, pero donde pagamos un peaje invisible. La paradoja se produce cuando perdemos el sentido de control y podemos ser la diana de aquellos que pueden reconocer tanto nuestro perfil como nuestra ubicación. Así pues, la pregunta que todo el mundo se debe hacer es: ¿acepto ser la moneda de cambio sin poder elegir cuándo y cómo quiero serlo? Un ejemplo reciente fue la noticia de una madre que perdió a su hijo mientras estaba embarazada y denunció en las redes el continuo bombardeo de anuncios de productos para niños. Mientras la futura madre navegaba por la red, la inteligencia artificial con la que están dotadas las redes publicitarias tomaba nota de su interés en productos relacionados con embarazadas, recién nacidos, etc., pero cuando desapareció este interés por la desafortunada pérdida del bebé, los algoritmos no fueron lo suficientemente inteligentes como para comprender el dolor que generaban los anuncios que aparecían en el móvil de esta mujer.

Es lógico que el mundo económico y las empresas que invierten aprovechen al máximo las posibilidades que les ofrece la tecnología móvil. Es algo lícito y muy goloso. Sin embargo, estas compañías pueden correr el riesgo de traspasar barreras éticas que hoy no están bien delimitadas por la novedad de esta disciplina.

Recientemente, una compañía líder mundial en mensajería instantánea ha implementado un plan piloto ${ }^{1}$, conjuntamente con un pool muy selecto de empresas anunciantes de todo el mundo, que consiste en el envío automatizado, instantáneo y personalizado, de mensajes de texto enviados directamente al móvil dentro de la cuenta de mensajería personal del usuario. Cuando una persona solicita información de un producto concreto a través de un formulario típico de web, el nuevo sistema crea un mensaje personalizado y lo envía directamente a la cuenta personal de mensajería instantánea que utiliza el usuario sin que este haya dado un consentimiento explícito y

1. Datos no referenciables por razón de confidencialidad profesional. 
creyendo que recibiría una simple llamada telefónica. La innovación no es la personalización ni la automatización del mensaje de respuesta, sino la instantaneidad y el canal donde se emite. El objetivo de la empresa anunciante, en el fondo, es poder elegir leads de calidad y solo responder los mensajes que el usuario contesta desde su móvil. Esto es muy importante para la rentabilidad de la empresa y de las campañas de captación de datos, ya que ahorra una gran cantidad de costes de personal comercial y administrativo, porque no es lo mismo atender indiscriminadamente miles de contactos a atender solo unos cientos. El hecho es que el usuario piensa que hay otra persona en el otro lado de la aplicación, cuando no es cierto. De hecho, ha sido un robot de inteligencia artificial que se identifica con un número de teléfono que no se puede contestar. La polémica, pues, acontece cuando una persona da sus datos y el receptor (el anunciante) los utiliza como y cuando quiere, como si fuera una patente de corso amparándose en el deseo del consumidor, entrando en su esfera personal, tal como es percibido el dispositivo móvil actualmente.

Otra alteración que está provocando la tecnología móvil, esta vez en beneficio del consumidor, es el efecto de los llamados ROPO y showrooming; es decir, la relación entre búsqueda de productos y su adquisición. En el primero de los casos, el usuario busca online y compra en la tienda física, y en el segundo, al contrario, busca en la tienda física y compra online. Internet está dando visibilidad a miles de productos y la información que puede obtenerse condiciona el comportamiento de los comerciantes, tanto a nivel de servicios como de precio. Este fenómeno ya existe desde hace dos décadas y es bien conocido por muchos expertos y estudiosos de la economía digital (Mróz Gorgon, 2018). Actualmente, está adquiriendo una dimensión muy relevante por la magnitud de las cifras del comercio electrónico y la crisis que ocasiona en aquellos comerciantes que no se están adaptando a la nueva realidad móvil. Hoy no se han identificado de forma clara los patrones de comportamiento de los usuarios en movilidad y, por ende, los vendedores no pueden actuar en consecuencia. Todo ello nos muestra una realidad compleja, y los pequeños vendedores, sin los recursos de las grandes firmas comerciales, deben hacer lo imposible para responder a las necesidades de sus clientes y resistir la presión omnicanal de los competidores. Una competencia que puede utilizar la inteligencia artificial, la realidad aumentada y los chatbot, recursos solo al alcance de una minoría privilegiada.

Según un estudio de IAB a nivel mundial, las compras online por medio de smartphones y tabletas han llegado al $75 \%$ de los internautas. De la totalidad de las compras efectuadas, un 31\% han sido hechas online mediante un teléfono inteligente o tableta, pero resulta llamativo el dato que afirma que, de este porcentaje, un $7 \%$ optó por comprar en la tienda física pagando con el móvil. Unos porcentajes que, a buen seguro, seguirán aumentando en pro de los móviles, por el incremento de su uso, la mejora de la seguridad de los sistemas de pago y la confianza creciente que demuestran las nuevas generaciones de usuarios.

\section{Conclusiones}

La movilidad es, pues, un factor que está incidiendo en la sociedad y en la economía de una manera evolutiva y a gran velocidad. En consecuencia, el marketing no puede estar al margen de esta realidad mutante y las empresas ya no tienen que preguntar si es necesario practicar el marketing móvil porque la respuesta es obvia. Si bien los patrones de comportamiento a nivel de movilidad digital no están definidos todavía, las compañías deben asumir el reto de invertir recursos desde la perspectiva móvil, que seguro les puede aumentar la competitividad por el conocimiento adquirido de sus clientes.

\section{Referencias bibliográficas}

ADOBE. (2018). «The next Mobile decade. A report on the state of mobile maturity, ten years after our lives changed forever» [estudio en línea]. <https://landing.adobe.com/en/na/products/experience-cloud/ctir-3861-mobile-study/index.html\#/report/0>. 
AIMC. (2017). «Infografia Resumen $19^{\circ}$ Navegantes en la Red» [estudio en línia]. AlMC. <https://www.aimc.es/ otros-estudios-trabajos/navegantes-la-red/infografia-resumen-19-navegantes-la-red/>.

BONI, D. (2018). «Tres líderes del marketing explicando cómo destacar con anuncios bumper de seis segundos» [artículo en línea]. Think with Google. <https://www.thinkwithgoogle.com/intl/es-es/canales-de-publicidad/ video/tres-lideres-del-márqueting-explican-como-destacar-con-anuncios-bumper-de-seis-segundos/?utm_ source=content -alert\&utm_medium =email\&utm_campaign=slb-twg-esp-01-10-2019\&utm_ content=cta\&mkt_tok=eyJpljoiWVdaaE9HRXhNamxsTkRoaSIsInQiOiJ6bGJ4a01cLOhoTGdUOVN3YTlj Uk02Sm1KVmxTNEZweHJoYWliSzlsaGpBeFISMEIDWk1hZDByN3RKallrMWlyK2hNbDJOcW5JbEsyMEltM2NOdksxU2toREI2am9IMXJhR2QOU2FpN295bHUrVWMyaVIURHVRa3NCUGJXaGd3QzkifQ\%3D\%3D>.

DRUCKER, P.F. (1999). «Beyond the information revolution» [artículo en línea]. The Atlantic. <https://www.theatlantic.com/magazine/archive/1999/10/beyond-the-information-revolution/304658/>.

FLORE, E. (2015). «5 Things You Can Learn From The Story Of WhatsApp» [artículo en línea]. Medium Corporation, The History of Grip. <https://medium.com/the-story-of-grip/5-things-every-founder-can-learn-from-thestory-of-whatsapp-b6496bc4f54d>.

FLORES, CH. (2018). «La estrategia digital de Zara para sortear la apocalipsis del retail» [artículo en línea]. El Economista. <https://www.eleconomista.es/negocio-digital/noticias/9083008/04/18/La-estrategia-digital-deZara-para-lograr-sortear-al-apocalipsis-del-retail.html>.

GOOGLE. (2018). «Prepárate para la indexación "dispositivos móviles primero”» [artículo en línea]. Google <https:// developers.google.com/search/mobile-sites/mobile-first-indexing >

IAB. (2017). «Estudio Anual Mobile Márqueting» [estudio en línea]. IAB <https://iabspain.es/wp-content/uploads/ estudio-mobile-2017-vcorta.pdf>.

INSTAGRAM TUTORIAL. (2018). «The History of Instagram» [artículo en línea]. Instazood <https://instazood.com/ the-history-of-instagram/>.

MRÓZ GORGON, B. (2018). "The impact of the ROPO effect in the clothing industry». Economics and Business Review. Vol. 4 (18), n. ${ }^{\circ}$ 3, págs. 24-35.

OBSERVATORIO NACIONAL DE TELECOMUNICACIONES Y LA SOCIEDAD DE LA INFORMACIÓN. (2018). «EStudio sobre comercio electrónico B2C» [estudio en línea]. ONTSI <https://www.ontsi.red.es/ontsi/es/content/ estudio-sobre-comercio-electr\%C3\%B3nico-b2c-edici\%C3\%B3n-2017>.

OBSERVATORIO NACIONAL DE TELECOMUNICACIONES Y LA SOCIEDAD DE LA INFORMACIÓN. (2018). «Las TIC en los hogares españoles» [estudio en línea]. ONTSI. <https://www.ontsi.red.es/ontsi/sites/ontsi/ files/LXOleadaPanelHogares.pdf>.

PÉREZ, J., et al. (2018). «La evolución de Internet en España: del Tesys a la economia digital» [estudio en línea]. Red.es <https://www.ontsi.red.es/ontsi/sites/ontsi/files/50\%20A\%C3\%B1os\%20de\%20la\%20Red\%20 de\%20Redes.pdf>.

REDACCIÓN. (2018). «Por qué compañías como Uber, WhatsApp o Amazon quieren convertirse también en gigantes de la publicidad online» [artículo en línea]. Puro Márqueting. <https://www.puromarketing.com/25/31383/ companias-como-uber-whatsapp-amazon-quieren-convertirse-tambien-gigantes-publicidad-online.html>.

RIVILLAS, F. (2018). «Anuncios que no son iguales si llueve que si hace sol» [artículo en línea]. Madrid: Vodafone Empresas. <https://www.youtube.com/watch?time_continue=3\&v=O6Spq2-tuTA>.

SALESFORCE. (2018). «Salesforce predicts that, for de firts times ever, more purchesase will be made with mobile phones during the holiday seasons than any other devices» [artículo en línea]. PR Neswire. <https://www.prnewswire.com/news-releases/salesforce-predicts-that-for-the-first-time-ever-more-purchases-will-be-madewith-mobile-phones-during-the-holiday-season-than-any-other-devices-300711112.htmls.

SHANKAR, V. ET AL. (2010). «Mobile Marketing in the Retailing Enviroment: Current Insights and Future Research Avenues». Journal of Interactive Márqueting. Vol. 24, n. ${ }^{\circ} 2$, págs. 111-120.

SHANKAR, V. ET AL. (2009). «Mobile Márqueting: A Synthesis and Prognosis». Journal of Interactive Márqueting. Vol. 23, n.ํ르. págs. 118-129.

SMART INSIGHTS. (2013). «The evolution of email márqueting [infographic]» [artículo en línea]. Smartlnsights. $<$ https://www.smartinsights.com/email-márqueting/email-communications-strategy/email-márqueting-evolution/>. 
Cita recomendada: FOLGUERA OBIOL, Xavier. Movilidad, aquello que lo transforma todo. Oikonomics [en línea]. Mayo 2019, no. 11. pp. 8-14 ISSN: 2339-9546. DOI: https://doi.org/10.7238/o. n11.1901

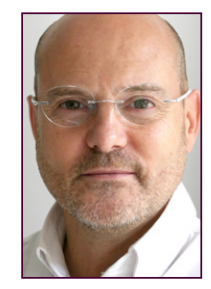

\section{Xavier Folguera Obiol}

xfolguera@uoc.edu

Profesor colaborador del máster universitario de Marketing Digital (UOC). Director de la agencia digital Advertis

Graduado en Diseño Industrial en la Escuela Superior de Diseño y Arte Llotja de Barcelona. Máster en Marketing Online y Comercio Electrónico por la Escuela de Administración de Empresas EAE/OBS. Actualmente desarrolla tareas docentes como profesor colaborador en la Universidad Oberta de Catalunya (UOC) en el Máster Universitario de Marketing Digital. Profesional con más de veinticinco años de experiencia como emprendedor, dirige la agencia de marketing digital Advertis, así como la plataforma de e-Commerce MINSPIRA, en el sector de la decoración y hogar. Es socio fundador del Colegio de Publicitarios y Relaciones Públicas de Cataluña.

Los textos publicados en esta revista están sujetos -si no se indica lo contrario- a una licencia de Reconocimiento 4.0 Internacional de Creative Commons. Puede copiarlos, distribuirlos, comunicarlos públicamente, hacer obras derivadas siempre que reconozca los créditos de las obras (autoría, nombre de la revista, institución editora) de la manera especificada por los autores o por la revista. La licencia completa se puede consultar en https://creativecommons.org/licenses/by/4.0/deed.es_ES.

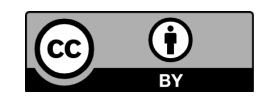

